\title{
Isotope Pattern Evaluation for the Reduction of Elemental Compositions Assigned to High-Resolution Mass Spectral Data from Electrospray Ionization Fourier Transform Ion Cyclotron Resonance Mass Spectrometry
}

\author{
Norbert Stoll and Enrico Schmidt \\ Institute for Automation, University of Rostock, Rostock, Germany
}

Kerstin Thurow

Center for Life Science Automation, Rostock, Germany

\begin{abstract}
The number of possible chemical formulae assigned to an accurate determined mass was significantly reduced by comparing spectral and theoretical isotope patterns based on mass measurement obtained with an ultrahigh-resolution electrospray ionization Fourier transform ion cyclotron resonance mass spectrometer (ESI-FTICR-MS) at high field intensity (7 T). Reduction is performed by rating congruency between experimental and theoretical pattern intensity and mass, and filtering out compositions with insufficient user-definable results. The methods used for isotope pattern simulation, peak searching, and comparison will be briefly described and evaluated on molecule ion signals of 25 compounds (300-1000 Da) applying a mass accuracy of $\pm 5 \mathrm{ppm}$, a set of eight elements with constant constraints $\left(\mathrm{C}_{0-200} \mathrm{H}_{0-1000}\right.$ $\mathrm{N}_{0-15} \mathrm{O}_{0-15} \mathrm{~S}_{0-2} \mathrm{Cl}_{0-2} \mathrm{Br}_{0-2} \mathrm{Ru}_{0-1}$ ), natural isotope abundances and experimental resolution (full width at half maximum). (J Am Soc Mass Spectrom 2006, 17, 1692-1699) (c) 2006 American Society for Mass Spectrometry
\end{abstract}

$\mathrm{D}$ etermination of elemental composition (EC) from high-accuracy mass measurement is a widely accepted method for gaining information on organic molecules determined in mass spectrometry [1, 2]. In theory, every distinct elemental composition has a unique mass that permits unique identification of a compound just from this exact mass [3]. In reality, accuracy in mass detection is limited, and more than one composition is usually found for molecules above $300 \mathrm{Da}$ - even with the high precision afforded by FTICR-MS over a wide mass range [4, 5]. In fact, the number of elemental compositions increases exponentially with mass, and many attempts have therefore been made to reduce the number of elemental compositions. Most of these are based on further chemical knowledge available for samples derived from other experiments and sample origins, and more constraint building blocks are used for combinatorial assignment instead of single elements; for example, amino acids or nucleotides are combined for large biomolecules [6, 7]. Even very complex mixtures of

Published online August 22, 2006

Address reprint requests to Dr. Enrico Schmidt, Institute for Automation, R.-Wagner Str. 31, Bldg. 8, D-18119 Rostock, Germany. E-mail: Enrico. Schmidt@uni-rostock.de compounds from refinery streams and humic acids have been successfully characterized by accurate mass measurement, taking into account the chemical relationship between constituents of mixtures and classification into a homologous series by Kendrick mass analysis [8-10]. The amount of a single element such as carbon can be determined by mass comparison of a ${ }^{13} \mathrm{C}$-enriched species against a species of the same compound that is naturally abundant, thus leading to a significant reduction in possible sum formulae [11].

High-resolution mass spectra contain a second informational dimension beyond the exact mass, peak intensity, or relative ion abundance. The spectral isotope distribution resulting from natural isotope abundance and elemental composition can be used to rate and exclude empirical compositions. Several methods have been developed to calculate isotope distributions of arbitrary composition with differences in efficiency and accuracy in mass and intensity [12-14]. Spectral properties such as resolution and system peak shape must be taken into account to obtain an accurate simulation. The intensity correlation between experimental spectra for the simulated isotope pattern of an elemental composition expressing the congruence between both patterns is determined and used to exclude elemental compositions with poor spectral representation. Use of this 
method for determining the content of bromine and chlorine in unknown substances and validating expected compounds from spectral data generated on a quadrupole instrument has been reported earlier. Recently, Kind and Fiehn described the identification of metabolites using this approach [16].

In this paper, we will demonstrate the application of the isotope pattern simulation and comparison method to high-resolution FTICR-MS mass spectral data. A known problem is that precision in intensity determination in organic mass spectrometry is no better than a few percent, and that additional difficulties occur in the determination of relative abundances of closely spaced cyclotron frequencies in FTICR-MS [17]. For this reason, the number of simulated isotopic peaks that have a spectral representation within the mass accuracy applied is used along with the intensity correlation to increase the filter effect. For nonoverlapped distributions, one would expect that the whole isotopic distribution would be determined with high mass accuracy, and the simulation should therefore not only match the isotope pattern in intensity, but also in exact mass expressed by the matched peak criteria.

\section{Experimental Materials and Methods}

\section{Samples}

A group of 25 synthesis products of known elemental composition within a mass range of 300 to 1000 Da was used for demonstration purposes. The synthesis products originated from catalysis and pharmaceutical research. The solvent was either a mixture of HPLC-grade isopropanol/water (50:50), acetonitrile/water (50:50) with $1 \%$ formic acid, or methanol/water (50:50) with $1 \%$ formic acid. Sample concentrations ranged from 1 to $2 \mathrm{mg} / \mathrm{ml}$.

\section{FTICR-MS+ Data Analysis}

Mass spectra were obtained using a commercial $7 \mathrm{~T}$ FTICR mass spectrometer (Apex III, Bruker Daltonik, Bremen, Germany) equipped with an external microelectrospray source. A $5 \mu \mathrm{l}$ sample was directly injected with a CTC-PAL autosampler (CTC, Zwingen, Switzerland) coupled with a syringe pump (Cole-Parmer Vernon Hills, IL) at a flow rate of $2 \mu \mathrm{l} / \mathrm{min}$ and sprayed at $20 \mathrm{lb} /$ in using a nitrogen nebulizer gas stream. In positive ion mode, the voltage between grounded sprayer needle and metal capped glass capillary entrance was $-4.5 \mathrm{kV}$ and $80 \mathrm{~V}$ to capillary exit. Transfer capillary was heated by nitrogen drying gas to $150{ }^{\circ} \mathrm{C}$. Prior detection ions were external accumulated within a hexapole ion guide for 0.3 to $0.6 \mathrm{~s}$, transferred to the analyzer cell and excited by frequency sweep pulse [4]. The system's mass range was set to $97,729-2000 \mathrm{Da}$, resulting in a sampling rate of $2 \times 1.111111 \mathrm{MHz}$. In broadband mode, $1024 \mathrm{k}$ data points were acquired, thus leading to a detection time of $0.472 \mathrm{~s}$ for a single

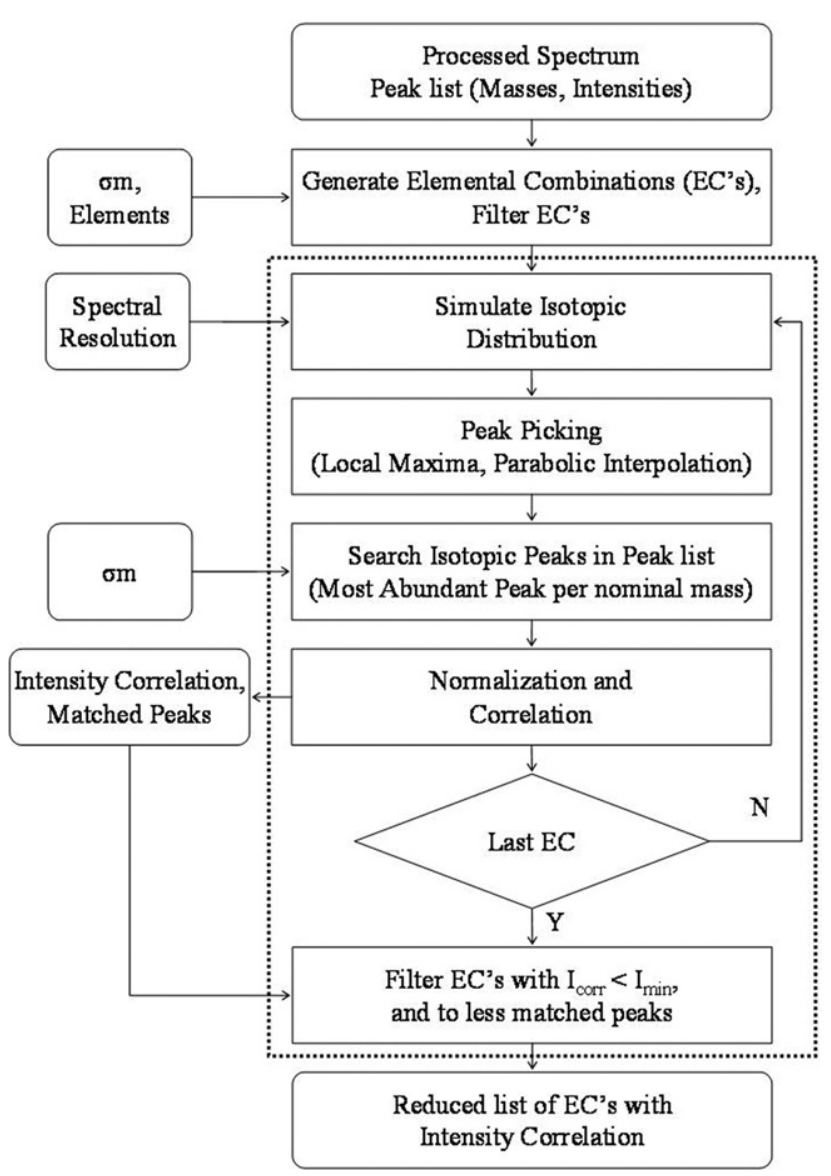

Figure 1. Flow chart of processes and input data applied to isotope pattern evaluation.

scan. Thirty-two single scans were summarized per spectrum. Analyzer cell pressure during detection was about $1.5 \times 10^{-10} \mathrm{mBar}$. After acquisition, mass spectral raw data were post-processed using XMASS acquisition and processing software (Bruker, Bremen, Germany). Data were apodized by multiplication with a halfperiod sine function zero-filled to $2048 \mathrm{k}$ data points, applied to Fourier transform and magnitude calculation. External calibration was performed before acquisition using single-charge caesium iodide clusters $\left(\mathrm{Cs}_{\mathrm{n}} \mathrm{I}_{(\mathrm{n}-1)}\right)^{+}$between $132.9(n=1)$ and 1172.1 $\mathrm{Da}(n=5)$ as reference masses, and a second-order polynomial as the calibration function between frequency and mass. Peak lists generated with XMASS as well as spectral resolutions according to FWHM criteria were taken as input data for calculations.

\section{Theory}

The information needed for the evaluation of high resolved mass spectral data consists of the exact masses and intensities for each peak (peak list), the set of elements to be considered including exact isotopic masses, abundances and valences, resolution for each peak, and absolute or relative mass accuracy ( $\sigma \mathrm{m}$ or $\sigma \mathrm{m} / \mathrm{m}$ ) [3]. Figure 1 summarizes the information flow 
and processes applied during the evaluation of a single peak from a spectrum. After generating elemental combinations for an accurate mass within a tolerance interval of $\pm \sigma \mathrm{m}$ as described elsewhere $[7,18,19]$, two filters are used to prescreen the list of empirical formulae and reduce the solution set before calculating the isotope pattern for each remaining $\mathrm{EC}$, and comparing the result with the experimental spectrum. The resulting correlation values are applied to a third filter that removes ECs with poor spectral representation.

The first filter calculates the number of rings and double bonds (double-bond equivalent, DBE) for an elemental composition referring to eq 1 , where $A_{1}-A_{6}$ are the amounts of atoms with valences of 1-6 [20].

$$
D B E=-\frac{A_{1}}{2}+\frac{A_{3}}{2}+A_{4}+\frac{3}{2} A_{5}+2 A_{6}+1
$$

A negative DBE expresses an oversized number of monovalent atoms in the EC that do not carry any chemical meaningful structural representation and therefore can be excluded from the list, with the exception of ECs with a DBE of -0.5 , which can occur due to addition of one hydrogen or other monovalent atom to a saturated compound $(\mathrm{DBE}=0)$ as happens during ES ionization, for example. Optionally, the filter function can be expanded by restricting the minimal and maximal DBE to user-defined values.

The second filter takes into account the anticipated ion state, which means the number of valence electrons in the composition. Neutral, nonradical compounds always have an even-numbered pair-wise arrangement of binding electrons signalized by an integer DBE value. In soft ionization techniques (ESI, APCI, FAB, MALDI), charge is often fed by adding or removing a monovalent ion such as $\mathrm{H}+, \mathrm{Na}+$, or $\mathrm{Cl}-$; the resulting $\mathrm{EC}$ of the molecule ion $\left(M+1 A_{1}\right)+$ will have an odd number of binding electrons and a fractional DBE (X.5); thus, ECs with integer DBE values or an even number of binding electrons can be removed from the solutions set for a supposed molecule peak if the corresponding ionization methods are applied.

The evaluation of the spectral isotopic distribution (Figure 1: dotted box) can be divided into two main parts: simulation of the theoretical spectrum of a sum formula and comparison between simulated and spectral patterns resulting in a correlation rating. The simulated isotopic distribution for an elemental composition $A_{a} B_{b} \ldots$ containing the elements $\mathrm{A}, \mathrm{B}, \ldots$ is determined by expanding the following expression

$$
\left(A_{1}+A_{2}+A_{3}+\ldots\right)^{a}\left(B_{1}+B_{2}+B_{3}+\ldots\right)^{b} \ldots
$$

where $A_{1}$ is the first isotope of element $A, B_{1}$ is the first isotope of element $B$, and $a, b, \ldots$ is the content of element $\mathrm{A}, \mathrm{B}, \ldots$ in the respective sum formula [12]. Each resulting unique permutation by the "polynomial" method represents one isotope configuration with the abundance
$P=\left(\frac{a !}{a_{1} ! a_{2} ! a_{3} ! \ldots} P_{A_{1}}^{a_{1}} P_{A_{2}}^{a_{2}} P_{A_{3}}^{a_{3}} \ldots\right)\left(\frac{b !}{b_{1} ! b_{2} ! b_{3} ! \ldots} P_{B_{1}}^{b_{1}} P_{B_{2}}^{b_{2}} P_{B_{3}}^{b_{3}} \ldots\right) \ldots$

where $a_{1}$ is the content, and $P_{A_{1}}$ is the abundance of the first isotope of element $\mathrm{A}$ in the isotope configuration, and the mass

$$
m=a_{1} m_{A_{1}}+a_{2} m_{A_{2}}+a_{3} m_{A_{3}}+\ldots+b_{1} m_{B_{1}}+\ldots
$$

with $m_{A_{1}}$ as the mass of the first isotope of element A. For efficiency reasons, only unique permutations for each element (referring to one term in a bracket in eq 3 ) are calculated separately. Pruning is also applied during this process, removing permutations with abundances smaller than a pruning threshold from further calculations, thus reducing calculation time and memory demands but introducing a slight distortion. Isotope configurations are formed afterwards by combining remaining unique permutations of the elements with each other. The result of the polynomial method is a list of isotopic peaks $\left(P_{i}, m_{i}\right)$ with infinite resolution. Many configurations have nearly the same exact masses and would overlap in an experimental spectrum due to the finite resolution of the mass spectrometer. To produce a representative simulation, isotopic peaks referring to a sum of unit impulse sequences $(\Delta(0)=1) I P$ $(m)=\sum_{i} P_{i} \Delta\left(m-m_{i}\right)=P_{1} \Delta\left(m-m_{1}\right)+P_{2} \Delta(m-$ $\left.m_{2}\right)+\ldots$ are convoluted with a peak shape function that should properly match the system's peak shape. The operator $*$ refers to the mathematical convolution defined by $f_{1}(t)^{*} f_{2}(t)=\int_{-\infty}^{\infty} f_{1}(\tau) f_{2}(t-\tau) d \tau$.

In FTMS, the peak shape approximates to Gaussian after apodization and, has therefore been selected (normalized to 1) for these calculations

$$
P S(m)=\exp \left(-\frac{m^{2}}{\sigma^{2}}\right)
$$

The unit impulse sequence behaves as a neutral element within the convolution integral, thus simplifying to a sum of peak shape functions with maximal intensity $\pi$ at mass $m_{i}$

$$
\begin{aligned}
I_{P}(m) & =P S(m) * I P(m)=\sum_{i} P S\left(m-m_{i}\right) P_{i} \\
& =P_{1} \exp \left(-\frac{\left(m-m_{1}\right)^{2}}{\sigma^{2}}\right) \\
& +P_{2} \exp \left(-\frac{\left(m-m_{2}\right)^{2}}{\sigma^{2}}\right)+\ldots
\end{aligned}
$$

Parameter $\sigma$, which complies with the standard deviation of the Gaussian function, is calculated with regard to the spectral resolution $\mathrm{R}=\Delta m_{50 \%} / \mathrm{m}$ (FWHM) at peak mass $m$. To equalize the spectral and simulated resolution, the peak width of the shape function at half maximum (0.5) must also be $\Delta m_{50 \%}$. Taking this pair of variates $\left(\Delta m_{50 \%} / 2,0.5\right)$ and inserting them into eq 5 
yields $\sigma=\Delta m_{50 \%}(-4 \ln 0.5)^{-0.5}$. The spectral resolution is assumed constant over the relative small mass range that an isotopic distribution occupies, and $\sigma$ is therefore a constant. For computational calculations, the desired mass range must be mapped to a number of data points, $\mu$. Let us assume that we have $\mathrm{w}$ data points within a mass area of $\Delta \mathrm{m}_{50 \%}$ with the data point density towards $\mathrm{w} / \Delta m_{50 \%}$. The mass range $\left(m_{\min }-m_{\max }\right)$ in the calculation is determined by the lightest and heaviest mass of simulated isotope configurations rounded down to the nearest integer and decremented $\left(m_{\min }\right)$ or incremented $\left(m_{\max }\right)$ by one. Because the heaviest and lightest isotope configurations of an EC depend on the pruning threshold set and the elemental composition itself, mass range varies and is recalculated for each simulation. Wider mass ranges are possible, but not necessary. The number of data points (NP) results from the product of data point density, and mass range and is rounded up to the nearest integer. Data point $\mu=0$ is mapped to $m_{\min }$ and data point $\mu=N P-1$ to $m_{\max }$, respectively. The mass on data point $\mu$ is therefore

$$
m(\mu)=m_{\min }+\mu\left(m_{\max }-m_{\min }\right) /(N P-1)
$$

The higher parameter $\mathrm{w}$ is set, the more precise the peak shape will be approximated and the smaller the spectral distortion introduced by discretization will be. On the other hand, the number of data points increases linearly with $\mathrm{w}$, as do calculation time and memory use. For the calculations presented here, $\mathrm{w}=7$ data points have been chosen since no significant changes in the results were observed using values above 7 . The resulting isotopic distribution over NP data points can be calculated using eqs 6 and 7. Due to the rapid drop in the Gaussian peak shape function, not every isotopic shape over the whole mass range $\left(m_{\min }<=m_{\mathrm{i}}<=\right.$ $\left.m_{\max }\right)$ needs to be computed. Within the interval of \pm 10 $\sigma$ around a center mass $m_{\mathrm{i}}$, the intensity falls to the $\exp (-100)=3.72^{*} 10^{-44}$-th part of peak intensity $\mathrm{P}_{\mathrm{i}}$; the calculation interval for a isotopic peak around $\mathrm{m}_{\mathrm{i}}$ can therefore be restricted to $\left(m_{\mathrm{i}}-10 \sigma\right)<=m_{\mathrm{i}}<=\left(m_{\mathrm{i}}+\right.$ $10 \sigma)$ without causing a significant error.

Peak picking must now be performed to obtain the resulting isotopic peak mass and intensity pairs out of the calculated array of NP data points; the local intensity maxima $\left[\mathrm{I}_{\mathrm{P}}\left(\mu_{\operatorname{lmax}-1}\right)<\mathrm{I}_{\mathrm{P}}\left(\mu_{\operatorname{lmax}}\right)>\mathrm{I}_{\mathrm{P}}\left(\mu_{\operatorname{lmax}+1}\right)\right]$ are determined, and the resulting maxima are interpolated by a second-order polynomial using the three points $[\mu$, $\left.\mathrm{I}_{\mathrm{P}}(\mu)\right]$ around a local maximum as grid points of the polynomial [21]. The respective data point is calculated setting the first deviation of the polynomial to zero; the maximum in intensity is found by solving the polynomial with this maximal data point. Other methods for finding the real top point from a peak shape are possible (weight average, simple maximum), and should be selected with regard to the method used with experimental spectra.
After simulation, the resulting isotopic peaks of an EC are searched within spectral data and the intensities are compared to each other. In this paper, only the most intensively simulated isotopic peaks per nominal mass have been considered and used for intensity correlation; possibly resolved isotopic fine structure therefore remains unevaluated. A mass window resulting from instrument mass accuracy around each remaining simulated peak is searched for spectral peaks. If no spectral peak, $i$, is located within this search window, the corresponding spectral intensity $I_{s}(i)$ is set to zero. In cases where more than one spectral peak is found inside the search window, the most intensive spectral peak is taken for correlation, as it should correspond to the most intensive isotopic peak that we are searching for, provided that both resolutions are equal (spectral and simulated) and no overlapping patterns occur.

To determine the intensity correlation, the intensities of the isotope pattern $I_{p}(i)$ are normalized to spectral intensities $I_{s}(i)$ by the least-square fit method, solving the first deviation of $\sigma^{2}=\sum_{i}\left(I_{S}(i)-\alpha I_{P}(i)\right)^{2}$ for $\sigma^{\prime}(\alpha)=$ 0 , yielding the normalization factor

$$
\alpha=\frac{\sum_{i} I_{S}(i) I_{P}(i)}{\sum_{i} I_{P}(i)^{2}}
$$

and intensity correlation $I_{c o r}$

$$
I_{c o r}(\%)=\left(1-\left(\frac{\sum_{i}\left(\alpha^{-1} I_{S}(i) I_{P}(i)\right)^{2}}{\sum_{i} I_{P}(i)^{2}}\right)^{0.5}\right) \cdot 100 \%
$$

as presented by Siegel et al. [15].

Each simulated isotopic peak that has a spectral representation $(\operatorname{Is}(i)>0)$ is marked as a matched peak. The isotope filter will remove all ECs with intensity correlation and number of matched peaks lower than user-defined values, thus reducing the remaining solution set.

\section{Results and Discussion}

The algorithms described have been implemented in the MassSpectral software package and used for the evaluation of the ESI-FTICR mass spectral data gained for 25 compounds of known elemental composition. Signals resulting from single charged molecule ions $\left((\mathrm{M}+\mathrm{H})^{+},(\mathrm{M}+\mathrm{Na})^{+}, \mathrm{M}^{+}\right)$were selected from peak lists. Element restrictions are chosen taking all compounds into account, which included hydrogen and carbon, both unlimited, oxygen and nitrogen ranging from 0 to 15 each, sulphur, bromine, and chlorine limited to a maximum of two and a maximum of one ruthenium, as there were five ruthenium complexes included. One sodium was required where the sodium abduct ion was evaluated. Elemental compositions were assigned for the mono isotopic peaks at a mass accuracy of $\pm 5 \mathrm{ppm}$, and only lightest isotopes of each 
element were combined. Double-bond equivalence was limited from -0.5 to 250 and the expected ion state according to molecule ion was used for filtering. The pruning threshold for isotope pattern simulation was set to $10^{-5}$. Minimal intensity correlation expected for an EC was $80 \%$; at least three simulated peaks had to be found in spectra for correct identification (matched peak criteria) in nonruthenium compounds. Due to the widespread isotope pattern of ruthenium, at least ten matches were expected for the complexes. A PC equipped with a $1.2 \mathrm{GHz}$ processor and $512 \mathrm{MB}$ RAM was used for calculation. Calculation time increased with the number of elemental compositions directed to isotope pattern evaluation, and ranged from some $\mathrm{ms}$ to $16 \mathrm{~s}$ for the heaviest compound with 612 ECs. The time taken forming and prefiltering the ECs was negligible, as it was always less than $80 \mathrm{~ms}$. Table 1 summarizes the calculation results for all compounds.

None of the proper sum formulae showed an intensity correlation beyond $80 \%$ and were thus eliminated from the solution set, and only three showed a correlation beyond $85 \%$. The reduction effect is shown in Figure 2. Requiring a minimal correlation of $80 \%$ and at least three matched peaks (ten for the last five compounds), the number of possible sum formulae could be reduced by $90 \%$ on average.

Figure 3 shows the measured and the simulated isotopic distribution of $\left(\mathrm{C}_{27} \mathrm{H}_{23} \mathrm{~N}_{4} \mathrm{O}_{3} \mathrm{~S}_{1} \mathrm{Br}_{1}+\mathrm{Na}\right)^{+}$ (Compound 15) at a resolution of 52,000. Intensity correlation was determined at $94.8 \%$, as there was no $100 \%$ optical congruity, and the spectral peak from nominal mass 589 Da did not lie within the mass window of 5 ppm around the simulated peak. Furthermore, simulated peaks from nominal mass 590 and 591 Da were not contained in spectral data, as they lay beyond the noise level and spectral intensities were consequently set to zero. From 121 elemental compositions assigned to measured mass 585.05,683 Da, only 16 showed an intensity correlation above $85 \%$ with at least three simulated peaks in the spectral data (Table 2).

For all of the 20 noncomplex compounds, the content of halogens in the remaining ECs was assigned exactly with the exception of Compound 14 containing two bromine atoms (Table 1), as ECs containing either $\mathrm{Cl}_{2} \mathrm{Br}_{1}$ or $\mathrm{Br}_{2}$ remained. The influence of $\mathrm{Cl}_{2} \mathrm{Br}_{1}$ and $\mathrm{Br}_{2}$ on the isotopic distribution was similar both in intensity and mass shift of isotopic peaks, where the resolution was not adequate to resolve isobar isotopic peaks resulting in similar isotope patterns; for example, the proper composition of Compound $14\left(\mathrm{C}_{22} \mathrm{H}_{26} \mathrm{~N}_{2} \mathrm{O}_{5} \mathrm{Br}_{2}+\mathrm{Na}\right)$ showed an intensity correlation of $92.3 \%$ with seven matched peaks, while an adequate correlation of $86.6 \%$ and six matched peaks was found for another composition from a solution set containing $\mathrm{Cl}_{2} \mathrm{Br}_{1} \quad\left(\mathrm{C}_{17} \mathrm{H}_{23} \mathrm{~N}_{6}\right.$ $\mathrm{O}_{6} \mathrm{Cl}_{2} \mathrm{Br}_{1}+\mathrm{Na}$ ). For the five halogen-free complexes, chlorine containing sum formulae remained, but these ECs had fewer matching peaks [10] within the spectral data than the actual compositions (Table 1).
Assuming sufficient intensity, the presence or absence of ruthenium was accurately detected since only elemental compositions including ruthenium had 10 or more isotopic peaks as required in the case of ruthenium containing compounds. Where ruthenium was not contained within a compound, compositions with ruthenium showed an intensity correlation beyond $80 \%$ and were also filtered out. As shown for Sample 15 in Table 2, the number of carbons could not be reliably estimated from ESI-FTICR spectral data since the abundance of carbon isotope $C^{13}$ was not high enough to indicate any change in intensity correlation beyond minimal correlation value with unsubstantial changing carbon numbers in the ECs [9]. Nevertheless, estimation was slightly better in nonhalogen and noncomplex substances as the carbon spread only ranged from -35.4 to $4.5 \%$ around the actual value, compared with -68 to $30 \%$ and -55.8 to $+22 \%$ for the complexes in halogenated compounds. Generally speaking, the higher the influence of an element (depending on amount and isotope abundances) to the resulting isotopic contribution is, the more accurately it will be detected, since it has more influence on intensity correlation. The isotope species of nitrogen and oxygen as well as of hydrogen only slightly contribute to the isotope pattern due to their low isotope abundances and / or low content and, thus, could not be estimated. The content of sulphur was only implicitly evaluated where the abundance of isotope configuration ${ }^{12} \mathrm{C}_{\mathrm{n}}{ }^{32} \mathrm{~S}_{\mathrm{c}-1}{ }^{34} \mathrm{~S}_{1}$ $(4.2 \%$ for $\mathrm{c}=1)$ was higher than contributions from other elements, such as the abundance of configuration ${ }^{12} \mathrm{C}_{\mathrm{n}-2}{ }^{13} \mathrm{C}_{2}{ }^{32} \mathrm{~S}_{1}$ (Compounds 1,2 , and $3, n<20$ ). In these cases, the presence of the $\mathrm{R}^{34} \mathrm{~S}$ isotopic peak, the third most abundant simulated nominal peak, was determined in spectral data and, if present, all compositions without sulphur were excluded (matched peak criteria). The peaks from the isotopic fine structure doublet in Figure 4 were nearly equal in intensity, and although two successive experimental spectra did show slight differences in intensity, peaks of the doublet could not be interchanged since the mass difference between $R^{12} C_{n-2}{ }^{13} C_{2}{ }^{32} S$ and $R^{12} C_{n}{ }^{34} S$ isotope configuration is about $11.7 \mathrm{mDa}$, sufficient for resolved isotopic peaks not to be interchanged at up to $1000 \mathrm{Da}$ given a $\pm 5 \mathrm{ppm}$ mass accuracy as they were not within the same search window. Commonly, the applied search and comparison algorithm excluded resolved but low-abundance isotopic peaks contributed by sulphur, and no sulphur content was detected (Table 2). Where the resolution was sufficient for separating ${ }^{12} \mathrm{C}_{\mathrm{n}-2}{ }^{13} \mathrm{C}_{2}{ }^{32} \mathrm{~S}$ and ${ }^{12} \mathrm{C}_{\mathrm{n}}{ }^{34} \mathrm{~S}$ configurations $(\mathrm{R}>\mathrm{m} / 11.7 \mathrm{mDa})$, the evaluation of isotopic fine structures was expected to increase the filter effect based on the matched peak criteria, as referring to Figure 4 for ECs with sulphur, there would be four spectral peaks detected instead of three.

If peaks contributed by sulphur were overlapped by, for example, carbon ${ }^{13} \mathrm{C}$ or bromine ${ }^{81} \mathrm{Br}$, only the high 
Table 1. Results for compounds investigated: spectral resolution measured FWHM ${ }^{\mathrm{a}}$, monoisotope mass of actual composition ${ }^{\mathrm{b}}$, number of elemental compositions without isotope pattern evaluation ${ }^{c}$, intensity correlation ${ }^{d}$, and number of matched peaks for actual composition ${ }^{\mathrm{e}}$, remaining compositions with correlation above 85 or $80 \%$ and relative reduction of amount of EC's related to $\mathrm{I}_{\text {cor }}>80 \%{ }^{\mathrm{f}}$. Note only in three cases intensity correlation of actual composition was lower than $85 \% \mathrm{~g}$

\begin{tabular}{|c|c|c|c|c|c|c|c|c|c|c|c|c|c|c|c|c|c|c|c|c|c|}
\hline \multirow[b]{2}{*}{ No. } & \multicolumn{8}{|c|}{ Sum formula } & \multirow[b]{2}{*}{ Resolution $^{a}$} & \multirow[b]{2}{*}{ Mol. ion } & \multirow[b]{2}{*}{$\Delta \mathrm{m}(\mathrm{mu})$} & \multirow[b]{2}{*}{$\begin{array}{l}\Delta \mathrm{m} / \mathrm{m} \\
(\mathrm{ppm})\end{array}$} & \multirow[b]{2}{*}{ Mex.(u) } & \multirow[b]{2}{*}{ Mthe. $(u)^{b}$} & \multirow[b]{2}{*}{$\# \mathrm{EC}^{\mathrm{c}}$} & \multirow[b]{2}{*}{$I_{\text {cor }}{ }^{d}$} & \multirow[b]{2}{*}{ \#Peaks ${ }^{\mathrm{e}}$} & \multirow[b]{2}{*}{$\begin{array}{c}\# I_{\text {cor. }}> \\
85 \%{ }^{f}\end{array}$} & \multirow[b]{2}{*}{$\begin{array}{c}\# I_{\text {cor. }}> \\
80 \%{ }^{f}\end{array}$} & \multirow[b]{2}{*}{$\begin{array}{l}\text { Reduct. } \\
(\%)^{f}\end{array}$} & \multirow[b]{2}{*}{$\begin{array}{c}\text { Calc. time } \\
(\mathrm{ms})\end{array}$} \\
\hline & $\mathrm{C}$ & $\mathrm{H}$ & $\mathrm{N}$ & 0 & $\mathrm{~S}$ & $\mathrm{Cl}$ & $\mathrm{Br}$ & $\mathrm{Ru}$ & & & & & & & & & & & & & \\
\hline 1 & 18 & 18 & 0 & 3 & 0 & 0 & 0 & 0 & 100,000 & $(\mathrm{M}+\mathrm{Na})^{+}$ & 0,452 & 1.5 & 305.115268 & 305.114816 & 5 & 90.82 & 3 & 1 & 1 & 80.00 & 60 \\
\hline 2 & 15 & 15 & 1 & 5 & 1 & 0 & 0 & 0 & 90,000 & $(\mathrm{M}+\mathrm{Na})^{+}$ & 0,13 & 0.4 & 344.056448 & 344.056316 & 18 & 88.5 & 4 & 1 & 2 & 88.89 & 130 \\
\hline 3 & 21 & 22 & 2 & 3 & 1 & 0 & 0 & 0 & 80,000 & $(\mathrm{M}+\mathrm{H})^{+}$ & 1,7 & 4.4 & 383.144066 & 383.142390 & 22 & 94.74 & 4 & 1 & 1 & 95.45 & 150 \\
\hline 4 & 20 & 20 & 2 & 2 & 1 & 1 & 0 & 0 & 80,000 & $(\mathrm{M}+\mathrm{H})^{+}$ & 1,3 & 3.4 & 387.094162 & 387.092853 & 32 & 87.6 & 4 & 4 & 5 & 84.38 & 170 \\
\hline 5 & 24 & 23 & 2 & 2 & 1 & 0 & 0 & 0 & 80,000 & $(\mathrm{M}+\mathrm{H})^{+}$ & 1,22 & 3 & 403.148692 & 403.147475 & 25 & 91.8 & 4 & 1 & 1 & 96.00 & 150 \\
\hline 6 & 28 & 28 & 2 & 2 & 1 & 0 & 0 & 0 & 70,000 & $(\mathrm{M}+\mathrm{H})^{+}$ & 0,92 & 2 & 457.195349 & 457.194425 & 29 & 96.4 & 5 & 2 & 2 & 93.10 & 180 \\
\hline 7 & 28 & 52 & 2 & 4 & 0 & 0 & 0 & 0 & 65,000 & $(\mathrm{M}+\mathrm{H})^{+}$ & $-0,8$ & -1.7 & 481.399182 & 481.399984 & 5 & 96.6 & 4 & 2 & 3 & 40.00 & 70 \\
\hline 8 & 28 & 26 & 4 & 3 & 1 & 0 & 0 & 0 & 60,000 & $(\mathrm{M}+\mathrm{Na})^{+}$ & 0,66 & 1.2 & 521.162429 & 521.161783 & 49 & 85.7 & 3 & 5 & 6 & 87.76 & 360 \\
\hline 9 & 27 & 23 & 5 & 5 & 1 & 0 & 0 & 0 & 52,000 & $(\mathrm{M}+\mathrm{H})^{+}$ & 1,2 & 2.2 & 530.150456 & 530.149267 & 73 & $83.71^{\mathrm{g}}$ & 3 & 5 & 7 & 90.41 & 670 \\
\hline 10 & 34 & 30 & 2 & 2 & 1 & 0 & 0 & 0 & 60,000 & $(\mathrm{M}+\mathrm{H})^{+}$ & 0,07 & 0.1 & 531.210153 & 531.210075 & 57 & 87 & 3 & 4 & 5 & 91.23 & 420 \\
\hline 11 & 28 & 26 & 4 & 4 & 1 & 0 & 0 & 0 & 60,000 & $(\mathrm{M}+\mathrm{Na})^{+}$ & $-0,06$ & -0.1 & 537.156633 & 537.156698 & 65 & $84.2^{\mathrm{g}}$ & 3 & 5 & 7 & 89.23 & 430 \\
\hline 12 & 27 & 23 & 4 & 3 & 1 & 1 & 0 & 0 & 59,000 & $(\mathrm{M}+\mathrm{Na})^{+}$ & 0,08 & 0.1 & 541.107242 & 541.107161 & 83 & 88 & 4 & 7 & 10 & 87.95 & 730 \\
\hline 13 & 36 & 32 & 2 & 2 & 1 & 0 & 0 & 0 & 57,000 & $(\mathrm{M}+\mathrm{H})^{+}$ & 0,13 & 0.2 & 557.225855 & 557.225725 & 63 & 92.2 & 3 & 2 & 4 & 93.65 & 540 \\
\hline 14 & 22 & 26 & 2 & 5 & 0 & 0 & 2 & 0 & 55,000 & $(\mathrm{M}+\mathrm{Na})^{+}$ & 2,6 & 4.5 & 579.012658 & 579.010070 & 140 & 92.3 & 7 & 19 & 24 & 82.86 & 1,500 \\
\hline 15 & 27 & 23 & 4 & 3 & 1 & 0 & 1 & 0 & 52,000 & $(\mathrm{M}+\mathrm{Na})^{+}$ & 0,2 & 0.3 & 585.056825 & 585.056646 & 121 & 94.8 & 4 & 16 & 21 & 82.64 & 1,330 \\
\hline 16 & 39 & 33 & 5 & 2 & 0 & 0 & 0 & 0 & 52,000 & $(\mathrm{M}+\mathrm{H})^{+}$ & $-0,34$ & -0.6 & 604.270359 & 604.268015 & 69 & 88.2 & 4 & 5 & 6 & 91.30 & 650 \\
\hline 17 & 40 & 36 & 2 & 2 & 1 & 0 & 0 & 0 & 47,000 & $(\mathrm{M}+\mathrm{Na})^{+}$ & 1,4 & 2.2 & 631.240331 & 631.238970 & 84 & 88 & 3 & 6 & 8 & 90.48 & 830 \\
\hline 18 & 48 & 49 & 5 & 3 & 0 & 0 & 0 & 0 & 43,000 & $(\mathrm{M}+\mathrm{H})^{+}$ & $-1,1$ & -1.5 & 744.389721 & 744.390816 & 104 & $80^{9}$ & 4 & 4 & 7 & 93.27 & 1,350 \\
\hline 19 & 44 & 50 & 2 & 9 & 0 & 0 & 0 & 0 & 37,000 & $(\mathrm{M}+\mathrm{H})^{+}$ & $-2,7$ & -3.6 & 751.359571 & 751.362280 & 127 & 90.4 & 3 & 11 & 16 & 87.40 & 1,800 \\
\hline 20 & 51 & 41 & 5 & 2 & 0 & 0 & 0 & 0 & 42,000 & $(\mathrm{M}+\mathrm{Na})^{+}$ & 0,9 & 1.1 & 774.342329 & 774.341461 & 129 & 92.9 & 3 & 9 & 13 & 89.92 & 1,950 \\
\hline 21 & 46 & 36 & 6 & 6 & 0 & 0 & 0 & 1 & 37,700 & $\mathrm{M}^{+}$ & 1,5 & 1.7 & 864.178194 & 864.176683 & 501 & 86.2 & 12 & 13 & 23 & 95.41 & 9,850 \\
\hline 22 & 50 & 44 & 6 & 6 & 0 & 0 & 0 & 1 & 35,000 & $(\mathrm{M}+\mathrm{H})^{+}$ & 1,3 & 1.4 & 921.248397 & 921.247108 & 523 & 90 & 10 & 16 & 18 & 96.56 & 11,300 \\
\hline 23 & 52 & 48 & 6 & 6 & 0 & 0 & 0 & 1 & 32,000 & $\mathrm{M}^{+}$ & 1,2 & 1.3 & 948.271810 & 948.270583 & 550 & 93.2 & 12 & 31 & 37 & 93.27 & 13,000 \\
\hline 24 & 54 & 52 & 6 & 6 & 0 & 0 & 0 & 1 & 32,000 & $\mathrm{M}^{+}$ & 1,2 & 1.3 & 976.303120 & 976.301883 & 564 & 88.1 & 12 & 20 & 39 & 93.09 & 14,000 \\
\hline 25 & 55 & 52 & 6 & 7 & 0 & 0 & 0 & 1 & 32,000 & $\mathrm{M}^{+}$ & 2,9 & 2.9 & 1004.299691 & 1004.296798 & 612 & 90.9 & 12 & 23 & 36 & 94.12 & 16,000 \\
\hline
\end{tabular}




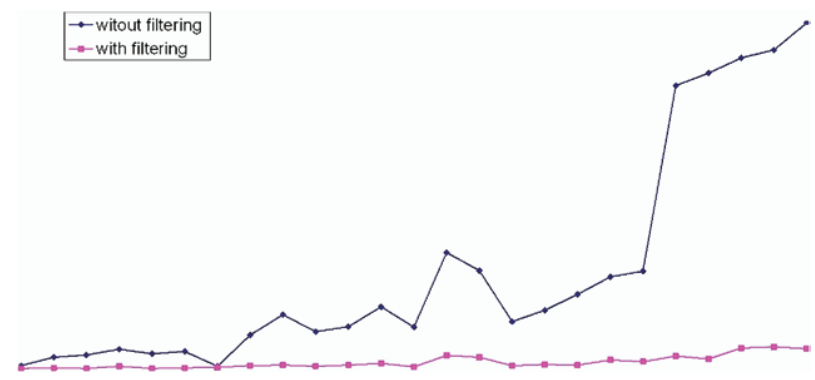

Figure 2. Number of elemental compositions without and with isotope pattern filtering.

content would have a significant influence on the isotopic distribution and, as in the cases of oxygen, nitrogen, and carbon, sulphur cannot be reliably detected, but it has been shown that isotopic fine structure contributed by carbon and sulphur can be resolved for proteins up to $16 \mathrm{kDa}$ with FTICR-MS [22].

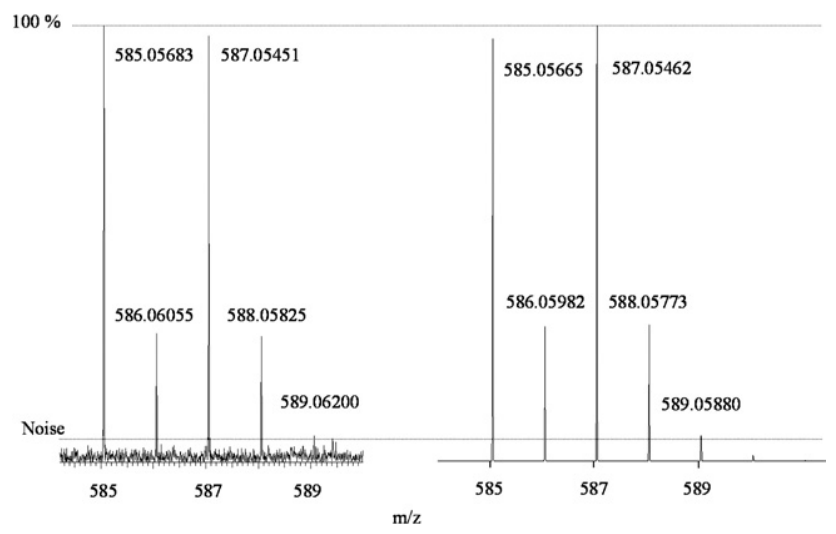

Figure 3. ESI-FTICR-MS (left) and simulated (right) spectrum of Compound $15\left(\mathrm{C}_{27} \mathrm{H}_{23} \mathrm{~N}_{4} \mathrm{O}_{3} \mathrm{~S}_{1} \mathrm{Br}_{1}+\mathrm{Na}_{1}\right)^{+}$.

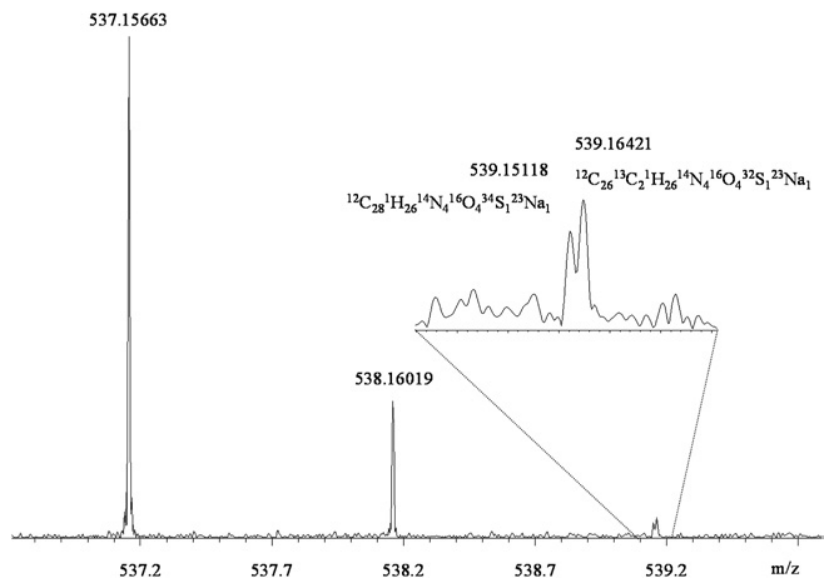

Figure 4. Resolved isotopic fine structure of Compound 11 $\left(\mathrm{C}_{28} \mathrm{H}_{26} \mathrm{~N}_{4} \mathrm{O}_{4} \mathrm{~S}_{1}+\mathrm{Na}_{1}\right)^{+}$at resolution 60,000 .

\section{Conclusions}

Isotope pattern evaluation led to an average relative reduction of elemental composition assigned to 25 compounds ranging from 300 to $1000 \mathrm{Da}$ of $90.6 \%$ and was thus found to be a valuable tool in the evaluation of high-resolution mass spectral data, although it was not possible to identify the content of each involved element accurately or to gain a unique composition since isotopic species of the elements involved such as hydrogen, oxygen, and nitrogen only have low natural abundances and therefore only a slight influence on the resulting pattern (or no influence if only one isotopic species exists). For example, the number of possible elemental compositions assigned to an ion at mass $751.35,957$ Da (Sample 19) and an experimental mass accuracy of \pm 5 ppm could be reduced from 127 to 11 . One requirement is sufficient intensity for obtaining a peak/information-rich distribution. A fixed set of eight elements $\left(\mathrm{C}_{0-200} \mathrm{H}_{0-1000} \mathrm{~N}_{0-15} \mathrm{O}_{0-15} \mathrm{~S}_{0-2} \mathrm{Cl}_{0-2} \mathrm{Br}_{0-2} \mathrm{Ru}_{0-1}\right)$ was

Table 2. Elemental compositions assigned to Compound 15 at mass 585.05683 Da with mass accuracy of 5 ppm, minimal intensity correlation of $85 \%$ and at least three isotope peaks recognized in spectral data

\begin{tabular}{|c|c|c|c|c|c|c|c|c|c|c|c|c|c|c|}
\hline \multirow[b]{2}{*}{ No. } & \multicolumn{8}{|c|}{ Sum formula } & \multirow[b]{2}{*}{$\mathrm{m}_{\text {the. }}(\mathrm{u})$} & \multirow[b]{2}{*}{$\Delta \mathrm{m}(\mathrm{u})$} & \multirow[b]{2}{*}{$\Delta \mathrm{m} / \mathrm{m}(\mathrm{ppm})$} & \multirow[b]{2}{*}{ DBE } & \multirow[b]{2}{*}{$\mathrm{I}_{\text {cor }}(\%)$} & \multirow[b]{2}{*}{ \#Peaks } \\
\hline & $\mathrm{H}$ & $\mathrm{C}$ & $\mathrm{N}$ & 0 & $\mathrm{Na}$ & $\mathrm{s}$ & $\mathrm{Cl}$ & $\mathrm{Br}$ & & & & & & \\
\hline 1 & 19 & 35 & 2 & 1 & 1 & 0 & 0 & 1 & 585.057297 & -0.000472 & -0.8 & 26.5 & 91.01 & 5 \\
\hline 2 & 15 & 20 & 14 & 2 & 1 & 0 & 0 & 1 & 585.0578 & -0.000975 & -1.7 & 19.5 & 95.17 & 5 \\
\hline 3 & 19 & 19 & 10 & 6 & 1 & 0 & 0 & 1 & 585.056464 & 0.000361 & 0.6 & 14.5 & 93.86 & 5 \\
\hline 4 & 23 & 23 & 4 & 8 & 1 & 0 & 0 & 1 & 585.05915 & -0.002325 & -4 & 13.5 & 97.29 & 5 \\
\hline 5 & 23 & 18 & 6 & 10 & 1 & 0 & 0 & 1 & 585.055128 & 0.001697 & 2.9 & 9.5 & 92.46 & 5 \\
\hline 6 & 27 & 22 & 0 & 12 & 1 & 0 & 0 & 1 & 585.057814 & -0.000989 & -1.7 & 8.5 & 95.98 & 5 \\
\hline 7 & 19 & 23 & 10 & 1 & 1 & 1 & 0 & 1 & 585.05396 & 0.002865 & 4.9 & 18.5 & 95.19 & 4 \\
\hline 8 & 23 & 27 & 4 & 3 & 1 & 1 & 0 & 1 & 585.056646 & 0.000179 & 0.3 & 17.5 & 94.82 & 4 \\
\hline 9 & 27 & 26 & 0 & 7 & 1 & 1 & 0 & 1 & 585.05531 & 0.001515 & 2.6 & 12.5 & 97.37 & 5 \\
\hline 10 & 23 & 11 & 12 & 8 & 1 & 1 & 0 & 1 & 585.055813 & 0.001012 & 1.7 & 5.5 & 85.87 & 4 \\
\hline 11 & 27 & 15 & 6 & 10 & 1 & 1 & 0 & 1 & 585.058499 & -0.001674 & -2.9 & 4.5 & 88.98 & 4 \\
\hline 12 & 31 & 14 & 2 & 14 & 1 & 1 & 0 & 1 & 585.057163 & -0.000338 & -0.6 & -0.5 & 87.52 & 4 \\
\hline 13 & 23 & 20 & 10 & 1 & 1 & 2 & 0 & 1 & 585.057331 & -0.000506 & -0.9 & 13.5 & 91.05 & 4 \\
\hline 14 & 27 & 19 & 6 & 5 & 1 & 2 & 0 & 1 & 585.055995 & 0.00083 & 1.4 & 8.5 & 90.37 & 4 \\
\hline 15 & 31 & 23 & 0 & 7 & 1 & 2 & 0 & 1 & 585.058681 & -0.001856 & -3.2 & 7.5 & 91.85 & 4 \\
\hline 16 & 31 & 18 & 2 & 9 & 1 & 2 & 0 & 1 & 585.054659 & 0.002166 & 3.7 & 3.5 & 89.46 & 4 \\
\hline
\end{tabular}


used for calculation (apart from sodium), as this could be used for automated interpretation of unknown compounds without user interaction. Filtering was based on intensity correlation and the number of simulated peaks found in spectrum within the same error interval $\pm \sigma \mathrm{m}$ used for combinatorial determination of empirical formulae. Further improvements are expected by evaluating the isotopic fine structure.

\section{Acknowledgments}

Funding from the German Federal Ministry for Research and Technology (BMBF), the Ministry for Education, Research, and Culture of Mecklenburg-Vorpommern, and the EU (Landesforschungsschwerpunkt grant no. UR 0201610 and CELISCA Center for Life Science Automation Technologies grant no. 6223001) is gratefully acknowledged.

\section{References}

1. Guan, F.; Soma, L. S.; Luo, Y.; Uboh, C. E.; Peterman, S. CollisionInduced Dissociation Pathways of Anabolic Steroids by Electrospray Ionization Tandem Mass Spectrometry. J. Am. Soc. Mass Spectrom. 2006, 17, 477-489.

2. Rodgers, R. P.; Blumer, E. N.; Freitas, M. A.; Marshall, A. G. Jet Fuel Chemical Composition, Weathering, and Identification as a Contaminant at a Remediation Site, Determined by Fourier Transform Ion Cyclotron Resonance Mass Spectrometry. Anal. Chem. 1999, 71, 51715176 .

3. Isotope masses and abundances are taken from: http://physics. nist.gov/PhysRefData/Compositions /

4. Marshall, A. G.; Hendrickson, C. L.; Jackson, S. J. Fourier Transform Ion Cyclotron Resonance Mass Spectrometry: A Primer. Mass Spectrom. Rev. 1998, 17, 1-35.

5. Marshall, A. G. Milestones in Fourier Transform Ion Cyclotron Resonance Mass Spectrometry Technique Development. Int. J. Mass Spectrom. Ion Processes 2000, 200, 331-356.

6. Pommerantz, S. C.; Kowalak, J. A.; McCloskey, J. A. Determination of Oligonucleotide Composition from Mass Spectrometrically Measured Molecular Weight. J. Am. Soc. Mass Spectrom. 1993, 4, 204-209.
7. Ioffe, B. V.; Vitenberg, D. A.; Zenkevich, I. G. Calculation of Ion Composition in Organic High-Resolution Mass Spectrometry. Org. Mass Spectrom. 1993, 28, 907-913.

8. Guan, S. Marshall, A. G. Resolution and Chemical Formula Identification of Aromatic Hydrocarbons and Aromatic Compounds Containing Sulfur, Nitrogen, or Oxygen in Petroleum Distillates and Refinery Streams. Anal. Chem. 1996, 68, 46-71.

9. Stenson, A. C.; Marshall, A. G.; Cooper, W. T. Exact Mass and Chemical Formulas of Individual Suwannee River Fulvic Acids from Ultrahigh Resolution Electrospray Ionization Fourier Transform Ion Cyclotron Resonance Mass Spectrometry. Anal. Chem. 2003, 75, 1275-1284.

10. Kendrick, E. A. Mass Scale Based on $\mathrm{CH}_{2}=14.0000$ for High Resolution Mass Spectrometry of Organic Compounds. Anal. Chem. 1963, 35, 2146-2154.

11. Rodgers, R. P.; Blumer, E. N.; Hendrickson, C. L.; Marshall, A. G. Stable Isotope Incorporation Triples the Upper Mass Limit for Determination of Elemental Composition by Accurate Mass Measurement. J. Am. Soc. Mass Spectrom. 2000, 11, 835-840.

12. Yergey, J. A. General Approach to Calculating Isotopic Distributions for Mass Spectrometry. Int. J. Mass Spectrom. Ion Phys. 1983, 57, 337-349.

13. Kubinyi, H. Calculation of Isotope Distribution in Mass Spectrometry. A Trivial Solution for a Nontrivial Problem. Anal. Chim. Acta 1991, 247, 107-119.

14. Rockwood, A. L.; Van Orden, S. L.; Smith, R. D. Rapid Calculation of Isotope Distributions. Anal. Chem. 1995, 67, 2699-2704.

15. Tong, H.; Bell, D.; Tabei, K.; Siegel, M. M. Automated Data Massaging, Interpretation, and E-Mailing for High Throughput Open Access Mass Spectrometry. J. Am. Soc. Mass Spectrom. 1999, 10, 1174-1187.

16. Kind, T.; Fiehn, O. Metabolomic Database Annotations Via Query of Elemental Compositions: Mass Accuracy is Insufficient Even at Less than 1 ppm. BMC Bioinformatics 2006, 7, 234-244.

17. Mitchell, D. W.; Smith, R. D. Cyclotron Motion of Two Coulombically Interacting Ion Clouds with Implications to Fourier-Transform Ion Cyclotron Resonance Mass Spectrometry. Phys. Rev. E 1995, 52, 43664386.

18. Robertson, A. L.; Hamming, M. C. MASSFORM: A Computer Program for the Assignment of Elemental Compositions to High Resolution Mass Spectral Data. Biomed. Mass Spectrom. 1977, 4, 203-207.

19. Dromey, R. G.; Foyster, G. T. Calculation of Elemental Compositions from High Resolution Mass Spectral Data. Anal. Chem. 1980, 52, $394-$ 398.

20. Pellegrin, V. Molecular Formulas of Organic Compounds: The Nitrogen Rule and Degree of Unsaturation. J. Chem. Educ. 1983, 60, 626-633.

21. Bronstein, I. N.; Semendjajew, K. A.; Musiol, G.; Mühlig, H. Taschenbuch der Mathematik; 3rd ed.; Verlag Harri Deutsch: Frankfurt am Main, Germany, 1997; p 854.

22. Shi, S. D.-H., Hendrickson, C. L.; Marshall, A. G. Counting Individual Sulphur Atoms in a Protein by Ultrahigh-Resolution Fourier Transform Ion Cyclotron Resonance Mass Spectrometry: Experimental Resolution of Isotopic Fine Structure in Proteins. Proc. Natl. Acad. Sci. U.S.A. 1998, 95, 11532-11537. 